\title{
Entre información y procesos de comunicación La inteligencia territorial ${ }^{*}$
}

\author{
Yann Bertacchini *
}

\section{Resumen}

Las perturbaciones que se producen en los territorios y las referencias espaciales como consecuencia de los cambios y transformaciones plantean problemas tanto a los políticos como a todo actor local, en la medida en que ellos funcionan sobre la delimitación territorial de sus competencias. Por lo tanto, tales perturbaciones no excluyen la interferencia de los territorios y no excluyen modos de coordinación más o menos invisibles. De este modo, el espacio invisible adquiere un peso creciente. Así, el espacio público se reduce cada vez menos a un espacio físico definido por calles y plazas; en tanto el espacio mediático a través de los debates que promueve juega un rol cada vez más importante.

En este artículo definiremos la inteligencia territorial como un proceso informacional y antropológico, regular y continuo, iniciado por actores locales, físicamente presentes y/o distantes, que se apropian los recursos de un espacio movilizando, y en consecuencia transformando, la energía del sistema territorial en capacidad de proyecto. De este modo, la inteligencia territorial puede ser asimilada a la territorialidad que resulta del fenómeno de apropiación de los recursos de un territorio, luego a las

\footnotetext{
* Artículo recibido 23 de abril de 2013. Aceptado 12 de septiembre de 2013.

Traducido por Ana María Pérez.

* Maître de Conférences, HDRInformation \& Communication. Expert près I'U. E. Université du Sud (Toulon-

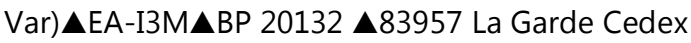

Contacto: bertacchini@univ-tln.fr
} 
transferencias de las competencias entre las categorías de actores locales de cultura diferente. El objetivo de este enfoque es asegurar, en sentido literal y figurado, en el nivel territorial las posibilidades para desarrollar lo que hemos denominado el capital formal territorial.

\section{Palabras clave}

Apropiación - capital - inteligencia - movilidad - observación.

\section{Abstract}

Due to globalization and whatever the field, major change implies as much as deep transformation of thinking i.e. to put in network new skills in particular, those made available by the availability of information and communication technologies. In this context, only one individual cannot join together all necessary skills. It becomes clear that the goal of space valorization cannot be considered without relational investment between the partners and their accumulated background. The local and territorial empowerment concerns a collective phenomenon of acquisition and development of skills rests on an learning way appropriate to the potential of the territory to form or promote. The local mediation sets for objective the creation of relations between pre-existent skills and their transfer between the actors, individuals or groups, private or public. But the capacity of the territory to solve these problems will depend closely on the richness of the information and communication process network and co-operations which it shelters or it will suggest.

\section{Keywords}

Information - local - networking - process - skill - territorial.

Aunque en su origen considerados físicos, los niveles territoriales integran progresivamente las TIC; desdibujando los límites administrativos y favoreciendo la emergencia de territorios virtuales. De este modo, la "sociedad de la información" se construye. En consecuencia, así como es primordial que los territorios integren estas tecnologías de la información y de la comunicación, es igualmente necesario que la nutran, a riesgo de encontrarse marginalizados tanto en un plano nacional como internacional. El proceso de inteligencia territorial, que puede ser calificado como desarrollo de la información territorial, encuentra aquí su plena justificación, contribuyendo a la constitución de capital formal a nivel territorial. Desde nuestra 
perspectiva, el capital formal de nivel territorial es el paso previo a toda política de desarrollo, se trate de política de cambio territorial, de reconversión o de innovación. Estos aspectos refieren, al menos en parte, a infraestructuras, redes de telecomunicaciones de banda ancha (tubos), las que, con el soporte y el contenido de los documentos digitalizados, son creadas gracias a estas herramientas. Estas cuestiones interesan a todos los sectores de la sociedad, de la educación a la economía, pasando por la salud; el mundo de la administración, el de la empresa e incluso lo particular.

Antes de avanzar, definiremos la inteligencia territorial como un proceso

informacional y antropológico, regular y continuo, iniciado por actores locales físicamente presentes o distantes, que se apropia de los recursos de un espacio movilizando y luego transformando la energía del sistema territorial en capacidad de proyecto. De este modo, la inteligencia territorial puede ser asimilada a la territorialidad que resulta del fenómeno de apropiación de los recursos de un territorio, igualmente, a las transferencias de las competencias entre categorías de actores locales de cultura diferente. El objetivo de este desarrollo es el de dotar al nivel territorial de capacidad para desarrollar lo que hemos denominado el capital formal territorial.

Presentamos estas características más adelante.

Desde el punto de vista de las $\mathrm{SIC}^{1}$, este proceso informacional tanto como antropológico supone la conjunción de tres hipótesis (Bertacchini, 2000):

- los actores intercambian información (energía);

- otorgan crédito a la información recibida (información):

- una vez el proceso de comunicación establecido, los actores establecen las redes apropiadas y transfieren sus competencias (proyecto).

Cuando estas hipótesis están reunidas y verificadas, el conjunto de competencias pueden ser identificadas con la ayuda de acciones de información y comunicación territoriales y luego movilizadas en la perspectiva del desarrollo. Para nosotros esto constituye un preámbulo para la definición de una política de desarrollo local de naturaleza endógena.

Sontenemos, además, que la inteligencia territorial se construye en torno de dos ejes fundamentales; sin mencionar el eje del tiempo que resta, cualquiera sea el objeto para estudiar y a fortiori cuando se trata de estudiar un territorio.

\footnotetext{
${ }^{1}$ Ciencias de la información y de la comunicación.
} 
La variable tiempo es inevitable, dado que un debilitamiento en ese proceso perturba la constitución del capital formal territorial. De hecho, podríamos evocar al respecto las diferentes líneas de tiempo que forman su eje general:

-el eje lateral, que comprende el territorio físico y cuyo objetivo es medir el potencial de acción local;

- el eje vertical, que organiza los vínculos y relaciones entre el territorio físico y sus representaciones virtuales.

En este sentido, la inteligencia territorial conjuga información y procesos de comunicación, aunque no puede reducirse a una acción de vigilancia territorial.

\section{La creación de contenidos territoriales deriva de procesos de comunicación}

A esta altura de la propuesta, nos proponemos asociar los actores locales que participan a su creación y a su difusión. Consideramos que los contenidos pueden derivar de prácticas institucionales, ciudadanas o económicas. Podemos citar como ejemplo las iniciativas de democracia local; las funciones de los territorios rurales, la valorización cultural y patrimonial; el contenido redaccional; la formación de los delegados, la creación de comunidades virtuales.

El desarrollo local reposa tanto en la creación de contenidos de carácter local como en portales y contenidos temáticos que contribuyen a asociar los conocimientos y experticias en ciertos campos de actividad o en temas de utilidad social. Se trata de asegurar la diversidad de los contenidos adaptados a las realidades y necesidades de los diferentes grupos y categorías de población y de puntualizar las ausencias o insuficiencias para la implementación y práctica de la inteligencia territorial.

Los objetivos asociados a los programas de creación de contenidos pueden ser formulados de la siguiente manera y atendiendo a estos principios: la formación y la participación de los actores locales en la creación de una riqueza colectiva y de saberes compartidos.

Los principios de base de los programas de creación y de desarrollo de contenidos deberían:

1. estar basados en estudios de necesidades por grupos y categorías (personas de edad, comunidades cualturales, sin empleo, jóvenes, discapacitados) ;

2. favorecer y mantener convocatorias orientadas a la creación de contenidos originales y la digitalización de los ya existentes sobre otros soportes (escrito, video, etc.) ;

3. sostener la confección de bases de datos y la difusión en línea de las innovaciones y de los contenidos, con normas de catalogación y motores de búsqueda especializados ; 


\section{Inteligencia territorial y Desarrollo}

En este punto, nos proponemos explicar cómo las TIC contribuyen a rediseñar las fronteras territoriales, e igualmente cómo y de qué manera la información y la comunicación territorial ayudan, o pueden ayudar, en la constitución del capital formal de un territorio. Los participantes de la creación de contenidos locales acuerdan que el territorio conocido ha sido rediseñado y la información y la comunicación han tenido, desde siempre, un rol determinante en la constitución y el desarrollo de los territorios. Actualmente, las TIC son suficientemente fuertes, y esto, aunque no se encuentren suficientemente repartidas en el conjunto de los niveles locales. Uno de los factores responsable de la desigual implantación geográfica de las TIC —aspecto que hemos, por otro lado, subrayado desde temprano en nuestro proyecto- es la constatada ausencia de gestión de los proyectos. En efecto, sin gestión transversal, no puede haber difusión del conocimiento ni desarrollo local fundado sobre los recursos de las TIC. Su implementación, en beneficio del desarrollo territorial, exige recurrir a competencias que son por esencia pluridiciplinares. Se trata, en consecuencia, de crear entornos de aprendizaje propicios al desarrollo territorial mediante la difusión de conocimientos múltiples. Es allí precisamente donde la capacidad —o la incapacidad- del territorio para obtener estos resultaados. Vamos a continuación a retomar algunos de estos aspectos con el fin de plantear las bases de nuestra propuesta.

\section{Una retrospectiva simplificada de la noción de complejo local}

Lo local es un sistema $y$, en tanto tal, resulta conveniente promover una gestión apropiada del proceso que permita que los actores locales vayan desde la idea a la creación de contenidos. Asimismo, en cuanto a la reflexión y definición del proyecto de desarrollo territorial, este no siempre es recordado o se ponen en perspectiva las sucesivas etapas que condujeron a los actores locales a interrogarse sobre el rol de soporte que brindan las TIC. Esta carencia constituye desde el inicio un error bastante perjudicial. No se puede abstraer a la inteligencia territorial de su desarrollo histórico. Nuestra práctica del territorio, en el sentido físico del término, no nos prepara adecuadamente para visualizar otras relaciones en su lugar. No podemos borrar los miles de años de prácticas territoriales, incluso si la velocidad de los desplazamientos y las transformaciones autorizadas han acarreado cambios irreversibles. Hemos destacado, desde los años 96-99, que lo local ha sido objeto de un redescubrimiento acompañado probablemente por la necesidad de constituir una identidad local. Este redescubrimiento y utilización de lo local ha sido favorecido y ampliado por el discurso sobre las TIC. Por lo tanto, hoy resulta legítimo preguntarse acerca del realismo ligado a la difusión y al empleo de ellas, en particular en relación con la creación de 
contenidos. En efecto, ¿se trata de un fenómeno de moda o de un comportamiento oportunista?

\section{Una cultura de la participación}

Los puntos de referencia que mencionamos a continuación traducen los rasgos de una cierta cultura participativa que remite a la dimensión gerencial y transversal del proyecto, a aproximaciones pluridisciplinarias y a numerosos prerrequisitos útiles para considerar. Si el proceso de creación de contenidos digitales no se fija como objetivo primero vincular a los ciudadanos, los empresarios, los administradores, los territorialistas, los educadores sociales, la política local de desarrollo no puede pretender edificar la organización agregativa de sus prácticas. Esta dimensión está hoy comprometida por la individualización de las prácticas, las iniciativas y por el uso individual de las TIC, que se han difundido en casi todos los sectores de la sociedad.

Cuando, por ejemplo, los actores de una organización, de una universidad o de una diplomatura solo consideran el componente TIC de su acción pedagógica en una propuesta de Educación a Distancia se equivocan; y desde nuestra perspectiva no producen un acto de desarrollo territorial en el sentido de la inteligencia territorial. En efecto, si el nivel local no es portador de esta cultura o no logra promover proyectos transversales, tal organización no podría en ningún caso detectar, formar y agregar las competencias. Así, la primera misión del proceso de inteligencia territorial incluye tanto la detección de competencias locales como la organización de su transferencia. Podemos, además, mencionar otros prerrequisitos importantes, los que van más allá de la sola y simple sensibilización de los delegados, esto es, su formación en relación con las características mencionadas anteriormente y las potenciales consecuencias de su ausencia en el territorio. En efecto, cuando los ciudadanos no se reconocen ni en los proyectos ni en los componentes y prácticas locales - sean históricas, culturales, o geográficas - de su territorio, no logran una identidad que se construya a la vez en motor y resultado de la misma.

Esta fractura — que hemos denominado digital - para designar los excluidos de las TIC puede, desde nuestra perspectiva, designar un comportamiento individualista, reforzado por la difusión no asociativa de estas TIC. Su empleo puede permitir la creación de un sitio de Internet que no remita necesariamente a la dimensión local, contribuyendo a separar a los telespectadores de su entorno territorial próximo. Este mismo usuario puede, muy bien, hacer registrar el mencionado sitio fuera de sus fronteras o bien albergarlo en un servidor de Canadá. Asimismo, establecer un diálogo con las comunidades virtuales distantes sin que sea necesario compartir un enraizamiento territorial. Numerosos ejemplos pueden ilustrar estos comportamientos desterritorializados. ¿Cuáles serían en este caso los beneficios y los retornos esperados de tal difusión de las TIC sin transversalidades físicas y virtuales? 


\section{Una metodología transversal de proyecto}

Una vez que los territorios logran ubicar y, posteriormente, anclar el modelo ascendente de desarrollo, o modelo de desarrollo local, que hemos sumariamente reseñado más arriba, se observa, con bastante frecuencia, que el llamado enfoque en red y este modelo de desarrollo son iniciados, a título personal, por personas que se reconocen a sí mismas en los valores de su territorio o que encuentran allí una identidad para consolidar, ya se trate de empresas, organismos profesionales o de poder público. A partir de este propósito, la principal preocupación de los responsables de proyectos transversales sustentados en la difusión y el empleo de TIC está en la posibilidad de asociación. Sin embargo, estos aspectos son con frecuencia ignorados, o peor aun, disociados, alejándose, fatalmente del objetivo de desarrollo local.

Como conclusión intermedia de este recorrido sumario, es necesario insistir, especialmente, en la noción de proceso. La difusión de nuevas tecnologías, su puesta en acción, su apropiación por actores locales diversos por su cultura requieren un modo de empleo apropiado, gestionado a mediano y largo plazo.

Se trata de un proceso de aprendizaje que se verifica en un entorno territorial favorable. En lo esencial, consiste en un proceso organizacional elegido por territorios que habrían tomado conciencia de la necesidad de asociar competencias que hay que localizar previamente. Pero el camino para alcanzar los primeros resultados es largo y pesado. En este punto, recordamos que el objetivo es actuar en beneficio de un objeto de muchos millares de años, esto es, un territorio que también acoge ocupantes que sustentan prácticas igualmente antiguas. De este modo, esta agregación de competencias se va a traducir en la definición de reglas y de procedimientos bastante lábiles y adaptables a la cultura de los actores locales que quieran asociarse. Es lo que se califica de praxis, es decir, una práctica compartida, ya sea colectiva o comunitaria, pero bien alejada de las prácticas individualistas admitidas por las TIC.

A continuación, y considerando que al comienzo del artículo hemos planteado la idea de proceso y de los principios que lo acompañan, intentaremos dar respuesta a una triple interrogación: de qué manera aquello que definimos como desarrollo local finalizado puede devenir en proceso; de qué manera puede fusionarse o bien sostenerse en ese territorio, en nuestro espacio. Recordemos, a continuación, cuáles son las vías de acceso al desarrollo territorial.

\section{Las claves de una política de desarrollo local}

Existe una primera modalidad de desarrollo calificado como desarrollo exógeno. En este cuadro, el territorio convoca las competencias, las riquezas, los recursos externos, al espacio que hay que desarrollar y procura implantarlos en sus fronteras. En otros términos, se trata de insertar competencias sobre un existente local. El marketing 
territorial nha emprendido esta vía. Existe otra modalidad de desarrollo que se califica de endógena. Nosotros situamos la inteligencia territorial desde esta perspectiva. En ella, el territorio procura limitar, por el lado de una aproximación cualitativa, las redes tales como existen sobre un territorio en un instante « $T$ ». Cuando esta necesaria acción de vigilancia, que calificamos como pasiva, se ha llevado a cabo y, además, ha podido identificar, observar e inventariar las competencias disponibles, la inteligencia territorial debe orquestarlas, organizarlas logrando que las competencias sean transversales e intercambiables.

Si los territorios resultan diferentes en relación con el aspecto físico, lo son también en relación con sus competencias internas y con su manera de identificarlas. Atendiendo a esta cuestión, un conjunto de competencias locales delimitadas e identificadas en el cuadro de unos proyectos participativos, acciones y procesos de escucha tenderán a concretar el proceso iniciado a medida que dichas competencias se intercambien. De este modo, conducir el desarrollo local contribuye a la formación de lo que hemos designado como capital formal.

\section{Una federación de competencias: el capital formal territorial}

Nos proponemos acá una doble cuestión. La primera nos incita a pensar que, globalmente, las TIC son accesibles al conjunto de niveles territoriales. La segunda nos lleva a peguntarnos ¿por qué ciertos territorios no logran concretar su política de desarrollo, en tanto que las TIC están globalmente disponibles y han sido presentadas como el elemento ideal en este campo?

Esta pregunta antecede al desarrollo local e igualmente la definición y la implementación de un enfoque de inteligencia territorial. A la luz de esta doble cuestión, podemos preguntarnos legítimamente. Observamos que ciertos territorios logran dotarse, o disponen, de facultades para concretar su política de desarrollo; mientras que otros no alcanzan a asociar estas competencias. Y esto aun luego de haber detectado y organizado este conjunto territorial. Finalmente, otros, y en el peor de los casos, se pauperizan.

Nosotros consideramos que hay probablemente un modo de desarrollo que hay que privilegiar cuando el nivel local se dispone a iniciar una política de desarrollo local. Este modo de desarrollo resulta legítimo, y puede argumentarse. Es que lo intentaremos hacer a continuación.

Pensamos, en otros términos, que proponer un modelo de desarrollo exógeno es mucho más oneroso que recurrir a un modelo de desarrollo endógeno; y este es un señalamiento pleno de buen sentido. Tenemos probablemente a nuestras puertas, casi dentro de nuestros muros, si me autorizan esta metáfora, las compentencias necesarias para el modelo de desarrollo que se ha elegido. 
En ningún caso se trata de replegarse sobre sí mismo o sobre su territorio, sino de concebir que disponemos probablemente de más competencias internas que las que imaginamos y de capacidades para definir en común escenarios futuros. Este propósito no excluye recurrir de un tiempo a otro a operaciones de marketing territorial. Pero no se trata de la misma línea de tiempo ni del mismo contenido terriorial o del mismo sentido de la colectividad. El enfoque de la inteligencia se acomoda mal a los grandes anuncios y a corto plazo, incluso si los calendarios políticos le dan cabida a estas prácticas.

El enfoque de inteligecia territorial impone casi una reacomodación que supone proponer el modelo de desarrollo endógeno, el que se basa en principios complementarios. La adquisición de esta modalidad de desarrollo no es definitiva y suscita multiples interrogaciones. Nosotros presentamos más abajo algunas, esenciales.

¿Es posible que actores locales muy diferentes en cuanto a su cultura y posibilidades de movilidad quieran integrarse para reencontrar el proceso que evocamos? Una vez que han comprendido la importancia de construir un futuro común, de implicarse, ¿están en condiciones de transferir sus competencias? Una brecha muy real separa el pasaje de la intención al compromiso. Y finalmente, ¿es que los actores aceptan transferir lo que puede aparecer como del dominio de la propiedad personal?

Destacamos que el reconocimiento entre actores locales pasa necesariamente por un compartir los mismos objetivos, sin lo cual, no podemos dar credibilidad, consciente o inconscientemente, a la información brindada por nuestros interlocutores. De este modo, tampoco resulta posible pensar en la apropiación de dicha información, y, por el contrario, cuando se solicite su restitución, en el cuadro de intercambios o comunicaciones futuras ningún plusvalor podrá ser añadido.

Acabamos de describir brevemente un sistema que no se obtiene naturalmente y que denominamos el capital territorial o gramática del territorio (Jakobson, 1963).

\section{Hacia un ensayo de definición del capital formal de un territorio y de sus implicaciones}

Como contrapartida de los argumentos que acabamos de proponer presentamos la primera implicación, con la forma de algunos elementos de respuesta.

Primera implicación: es en el interior de la misma organización local donde se determinan las desigualidades de acceso a los territorios, al desarrollo o a la formulación de su proyecto.

Si aceptan la hipótesis que sostiene que un territorio puede recurrir a un modo de desarrollo endógeno, es decir, que el proceso de desarrollo puede ser iniciado a partir y con la ayuda de competencias internas locales, será necesario, en un momento u otro, medir estas competencias locales y sus capacidades para transferir. En consecuencia, por deducción lógica es posible proyectar que si nosotros somos débiles, 
territorialmente hablando, si no llegamos a desarrollarnos es probablemente debido a que nuestras reservas de competencias locales no son suficientes o no es posible conectarlas.

En última instancia se perfila la primera acción de un enfoque de inteligencia territorial, a saber, la detección de las competencias locales disponibles y los circuitos de información que ellas emplean.

\section{Segunda implicación}

Esta implicación deriva de la primera y se escinde en dos partes complementarias. El enunciado de la primera refiere precisamente a implicar o reconocerse a los actores locales en las acciones locales llevadas por las instancias. La segunda implicación destaca una transición importante en la actitud de estos mismos actores, y se propone promover intercambios, movilizarse y agruparse en torno del proyecto territorial. Esta implicación remite a la noción de compromiso, esto es, reconocerse en una identidad, pero también reconocerse en el código o valor de los actores locales con quienes intercambian información

\section{La condición es directa y reposa sobre el buen sentido}

¿Cómo pasar de la implicación a la capacidad de transferencia y de intercambio si no se le atribuye ningún crédito a la persona con quien se ha mantenido el intercambio? De esto trata la segunda implicación o segunda hipótesis. Si un actor no atribuye crédito a la información que recibe como devolución, no emitirá información creíble y los fundamentos de su enfoque de inteligencia territorial no existirán. Del mismo modo, los actores locales no les otorgarán ningún crédito a las informaciones cruzadas. ¿Cómo construir un modelo de desarrollo endógeno si hay carencia efectiva o al menos insuficiencia, en ese dominio o campo? Si por el contrario -y eso, felizmente, se produce - se acredita como válida y se acepta la información intercambiada, emitida y transmitida, se puede visualizar la cooperación, la coordinación de los actos de desarrollo. Este juego de actores que llamamos de crédito-crédito va a suscitar interacciones. Pero la partida está lejos de ser ganada, y, por lo tanto, nos queda formular otras dos implicaciones. Pero el enfoque de la inteligencia territorial se inserta bien en el proceso de comunicación.

\section{La tercera implicación}

El punto de partida de nuestra propuesta ha consistido en la formulación de este principio: podemos reconocer que para desarrollarse un territorio elije un modo de 
desarrollo endógeno. En consecuencia, el territorio va a ocuparse en acumular el capital necesario a su desarrollo, al que se denomina capital formal.

Se define al capital formal como sigue: se trata de un conjunto de reglas y procedimientos comunes. Cuando los actores locales aceptan compartir estas reglas y procedimientos, han tomado conciencia de que son adaptables a los miembros de la organización territorial y están dispuestos a afrontar la cuarta implicación, la que se encuentra en el corazón de muestra problemática y de nuestra investigación, a la que denominaremos inteligencia territorial.

Porque un nivel espacial o una organización territorial no podrían, o deberían, recurrir a una herramienta de asociación e iniciar este enfoque participativo antes de comenzar una política de desarrollo: un equipo de inteligencia territorial o un dispositivo sociotécnico de información y de mediación local.

Hasta el momento hemos intentado presentar la noción de inteligencia territorial, vamos a continuación a definir en qué consiste, diferenciándola de la inteligencia económica, noción que desde nuestra perspectiva resulta muy restrictiva o demasiado aplicada a los actores económicos y que, en consecuencia, no produce ninguna información específica. Por el contrario, el objeto de investigación, el territorio y el enfoque que proponemos, conjuga información y antropología. La inteligencia territorial descansa sobre un conjunto de actores muy diferentes entre sí y cuyos objetivos últimos no siempre se orientan a obtener resultados económicos, ni tampoco -o no siempre - se proponen una construcción de futuro con el mismo compromiso, aun cuando puedan ser evocados evocados en diferentes fases del desarrollo.

\section{El programa "MAINATE"}

A continuación, presentamos los trabajos que han sido realizados en el marco de este programa de investigación desarrollado en el seno del Laboratorio LePont. Mainate significa "Gestión de la información aplicada al territorio". Veamos a continuación estos objetos, estas primeras deducciones, estas primeras enseñanzas.

\section{El objeto o los objetos del programa "Mainate"}

Este programa se estructura en dos planteos que remiten a los dos modos de desarrollo evocados más arriba. Refiere a una metodología y a herramientas funcionales adaptadas a un modo de estrategia, el desarrollo territorial endógeno. Se interesa en la problemática de desarrollo enunciada, definida por los niveles de dimensión media, a los que han recurrido las TIC para construir su atractivo.

Este enfoque de composición del capital formal no supone, como ya lo hemos mencionado, una réplica local débil que consistiría en aislarse del mundo exterior. Teniendo en cuenta la esencia misma y el uso de las TIC, este esfuerzo en el interior de 
estructuración del capital formal va a devenir y ser empleado en un momento u otro, en un medio o vía para reforzar el atractivo del territorio.

Il s'agit du volet exogène du développement local dont l'objectif va consister en la diffusion de l'information à l'extérieur du complexe local. Après avoir reconnu en interne les compétences mobilisables localement, il faudra les faire connaître et reconnaître, les faire valoir. La démarche d'intelligence territoriale peut être assimilé à un repérage fantastique des savoirs locaux. Donc, le programme Mainate se décline en deux actes, de l'interne vers l'externe.

Se trata de un planteo exógeno del desarrollo local cuyo objetivo va consistir en la difusión de la información en el exterior del complejo local. Luego de haber reconocido en el interior las competencias movilizables localmente, será necesario hacerlas conocer y reconocer, hacerlas valor. El enfoque de inteligencia territorial puede ser asimilado a una delimitación fantástica de los saberes locales. En consecuencia, el programa Mainate se despliega en dos actos, de lo interno hacia lo externo. En conclusión: ¿dónde y cómo posicionar un enfoque de inteligencia territorial?

En un proceso de desarrollo local, la primera etapa consiste en la realización del diagnóstico estratégico del territorio y destacar sus fortalezas y debilidades. La propuesta de inteligencia territorial puede ser en consecuencia asociada a este diagnóstico estratégico. Pero, más que un diagnóstico estratégico, en sentido clásico del término, la perspectiva de inteligencia territorial pretende transformarse en una formidable propuesta pedagógica desarrollando la capacidad del territorio para desencadenar acciones cooperativas entre empresas, laboratorios, asociaciones. Como precisamos anteriormente, la inteligencia territorial se orienta a promover la articulación entre actores locales que no comparten los mismos objetivos y los mismos códigos. Consideramos también que, tanto la cultura como el patrimonio constituyen elementos clave del desarrollo. Son formidables palancas de desarrollo. Del mismo modo, el desarrollo de actividades y la puesta en red.

De la conducta del programa de investigación MAINATE, hemos podido identificar las etapas o momentos de lo que denominamos un metamodelo. Elegimos esta expresión porque es siempre difícil nombrar un modelo cuyo límites no han sido establecidos y cuando el objeto estudiado, el territorio, se destaca por su complejidad.

Primer momento: luego que el diagnóstico estratégico del territorio, corresponde llevar adelante una acción que permita identificar a los actores clave implicados en él, a título individual.

Segundo momento: con referencia a las hipótesis iniciales destacamos la importancia de reconocer la validez de la información recibida e intercambiada, porque de estos intercambios nacen las redes. Sobre un territorio, estas redes pueden existir, o no, y la inteligencia territorial, en relación con los aspectos que ella implica, se propone generar una dinámica de comunicaciones locales. 
Esta dinámica se naturaliza con la forma de interacciones. Este análisis permitirá precisar que en una determinada categoría de actores locales se detecta - o no- una carencia en sus intercambios. Se señalarán competencias y transferencia de competencias. Esta acción, probablemente, genere interacciones e incluso reacciones. Cuando se logra una agregación de acciones individuales y a posteriori, colectivas, por vía de interacciones, se produce un cambio de nivel. Vamos a inventariar los enfoques y las acciones en red localizadas previamente durante el período de observación realizado.

Desde una acción, estática, de indagación acerca de la existencia del territorio, nos interesaremos, a continuación, y desde un plano dinámico, en la puesta en red de portadores de proyectos. Vamos a interesarnos, sobre un plan dinámico, a la puesta en red de portadores de proyectos. Este enfoque produce interacciones locales. Y a continuación se busca lo que denominamos el plan de información, es decir, la concretización de intercambios de información en acciones que benefician el desarrollo territorial. Las acciones de inteligencia territorial respetan un orden: constatar, desarrollar, suscitar, detectar la ausencia o la presencia de interacciones y de proyectos de desarrollo. Pero, cuando se ha detectado una ausencia de este nivel sobre un territorio, raramente se logra formular un proyecto de desarrollo.

Habiendo presentado el metamodelo, nos interesa, en este punto, considerar otro concepto: la observación de las características del territorio en Internet. Lo designamos así porque nuestro objetivo consiste ajustarnos a la capacidad de desarrollo de los territorios y ciudades medias que ponen en acción las TIC como palancas de desarrollo.

Hemos identificado dos dimensiones:

1. dimensiones laterales: la referencia al territorio físico;

2. dimensión vertical: el prolongamiento virtual del territorio físico.

Dimensiones laterales: tal como ha sido indicado en este metamodelo.

Desde la perspectiva interna: ¿cuáles son los recursos en cuanto a competencias y capacidades de transferencia de estas competencias?, ¿cuáles son las fuerzas de cohesión internas en el proyecto?

Desde la perspectiva externa: se trata del otro planteo del enfoque de inteligencia territorial. ¿De qué manera vamos a reforzar nuestros atractivos? ¿Cuáles son las fuerzas de atracción externas de nuestro territorio? Esto hace referencia a la dimensión lateral.

\section{Dimensión vertical:}

Es interesante observar en Internet que un territorio intenta promoverse con la ayuda de un sitio, luego de detectar que el servicio que acoge el sitio de una ciudad, o de un territorio está albergado en Los Ángeles o en París o en Viena. Si el dispositivo no es en sí mismo contradictorio, es revelador de la capacidad —o de la incapacidadde ese territorio para pensar su desarrollo según la concepción de inteligencia 
territorial. De hecho, disponemos de herramientas que van a permitirnos representar el espacio virtual. Es decir, ¿de qué manera un internauta algo advertido percibe un territorio durante una navegación en Internet? Vía la ayuda de algunas herramientas de representación cartográfica y deduciendo la existencia, o la ausencia, de un territorio sobre Internet.

A continuación, desarrollaremos dos categorías de indicadores:

- físicos: nos referimos a los componentes geográfico-históricos.

- virtual: hacemos referencia a la presencia de un espacio territorial en esta representación cartográfica que se percibe con la forma de bits.

Podemos mencionar como ejemplo de un enfoque cualitativo de definición de indicador el análisis de redes que hemos practicado sobre distintos territorios, evaluando cuáles eran las conexiones entre los actores locales. Hemos materializado cuáles eran los lazos y las distancias entre estos actores locales. Se trata de la aplicación de un dispositivo sociotécnico local. Posteriormente hemos convocado a los actores locales con el fin de compartir con ellos nuestros resultados. Con ellos hemos detectado, por ejemplo, un conjunto de actores locales que declaran intercambiar informaciones entre ellos, configurando una suerte de mixtura de contactos entre actores diferentes.

En consecuencia, podemos deducir que nuestra hipótesis de desarrollo endógeno y el enfoque en red no puede encontrar aplicación sobre el territorio estudiado. Luego, por comparación, por procesos iterativos, comparativos, hemos aislado las debilidades locales. Para ir más lejos en la aplicación de nuestro análisis, podemos encontrar este tipo de cuestionamiento por parte de una ciudad, de un Consejo Regional, de un Consejo General. Y mediante la introducción de este dispositivo podemos intentar detectar las políticas anunciadas en materia de tecnología de la información y de la comunicación. Igualmente procurar clasificar el territorio en relación con otras ciudades, otras regiones, otros países.

Il s'agit donc bien entendu d'applications avec Internet. Du repérage du fournisseur jusqu'à l'étude de ce qu'on appelle, de ce que l'on nomme la littérature grise des thèses, des rapports, l'accès aux bases de données, des études économiques, d'évaluation d'un territoire. Il s'agit aussi bien entendu de repérer des acteurs publics avec une sensibilité voisine de façon à s'étalonner, à se comparer à d'autres territoires, et d'essayer d'être attentifs à leurs initiatives.

Se trata de aplicaciones con Internet. Desde la identificación del proveedor hasta el estudio de la llamada literatura gris proveniente de tesis, informes, accesos a bases de datos, estudios económicos, de evaluación de un territorio. Se trata también de identificar actores públicos con una sensibilidad que permita examinar, clasificar, compararse con otras próximas vecinas de manera de examinar, compararse con otros territorios, procurando estar atento a sus iniciativas. 


\section{BIBLIOGRAFÍA}

Atlan, H. (1986). A tort et à raison. Paris: Seuil.

Audretsch, D. B. (2001). Globalisation and communication technologies and the Role of Small firms in Innovation, in Feldman M. et Massard N., Institutions and systems in the geography of innovation, Kluwer Academic Publisher.

Bailly \& al, (1995).Collectif, Stratégies spatiales: comprendre et maitriser l'espace. Montpellier: GIP reclus.

Beauchard, J. (2003). La Mosaïque territoriale. Enjeux identitaires de la décentralisation. Paris: Éditions de l'Aube, Bibliothèque des territoires, $182 \mathrm{p}$.

Bertacchini Yann, (2012). Between information and communication process, the territorial intelligence, as a network concept \& a framework to shape local development. International Journal of Humanities and Social Science, IJHSS, ISSN 2220-8488 (Print), 2221-0989 (Online), Vol. 2 No. 18 Louisville KY 40202, U.S.A, September.

Bertacchini Yann, Maurel Pierre, Deprez Paul (2012). Réseaux \& Savoirs locaux: une médiation à organiser dans une démarche d'intelligence territoriale. 4ème édition des journées Management des Technologies Organisationnelles Workshop MTO'2012, Impact des réseaux numériques dans les organisations?, octobre 2012 Ecole des mines d'Alès - Site Georges Besse à Nîmes, Paris: Presses de l'école des mines

Bertacchini, Y. (2000) How to federate some local resources by developing new links?, Proceedings of ISA 23 Conference Rio de Janeiro, USA: The Endless Transition, Sciences Studies.

Bertacchini, Y. 2002b, Intelligence territoriale. Volet 1. Territoire et territorialités, septembre 2002, Toulon : Presses Technologiques, Collection Les ETIC.

Bougrain, F. (1999). Les enjeux de la proximité institutionnelle lors du processus d'innovation. Bordeaux: Revue d'économie régionale et urbaine N. ${ }^{\circ}$, IERSO.

Jakobson, R. (1963). Essais de linguistique générale. Paris: Editions de minuit

Jodelet, D. (1997). Représentations sociales: un domaine en expansion, en «Les représentations sociales». Sociologie d'aujourd'hui, Paris: PUF.

Kant, E. (1912). Critique de la raison pure. Traducido del aleman por Jules Barni, corregido por P. Archambault.-Paris, Paris: E. Flammarion (1912). 2 vol In-12 ${ }^{\circ}$, [8 ${ }^{\circ}$. 25527], (Les meilleurs auteurs classiques français et étrangers).

Major, W. (1999). Approche systémique du territoire. Colloque de la Systémique, Universidad de Zurich.

Poche, B. (1996). L'espace fragmenté. Eléments pour une analyse sociologique de la territorialité, Paris: L'Harmattan.

Porter, M. E. (1990). Europe's Companies After 1992: Don'tCollaborate, Compete.The Economist, June 6, pp. 17-19.

Prax, J-Y (2001).Le management territorial à l'ère des réseaux, Paris: Editions d'Organisation, Dunod. 
Prelaz-Droux, R. (1995).Conception d'un système d'information à référence spatiale pour l'aménagement et la gestion du territoire, Approche systémique et procédure de réalisation. Lausanne, Suisse : EPFL

Schwarz, E (1997).Toward a Holistic Cybernetics. From Science Through Epistemology to Being. Alborg: Cybernetics and Human Knowing, Vol. $4 \mathrm{n}^{\circ} 1$

Tapscott, D, Agnew D,(2000). Quelle gouvernance pour l'économie numérique?Problèmes économiques $\mathrm{n}^{\circ} 2.675$, Paris : Economie Internationale.

Vodoz, L et al, (2002).NTIC et Territoires, enjeux territoriaux des NTIC, Lausanne: Presses Universitaires Fédérales Suisse Romande. 